\title{
Quantification of the Intracellular Life Time of Water Molecules to Measure Transport Rates of Human Aquaglyceroporins
}

\author{
Madelene Palmgren $^{1} \cdot$ Malin Hernebring $^{2} \cdot$ Stefanie Eriksson $^{3} \cdot$ Karin Elbing $^{2} \cdot$ \\ Cecilia Geijer $^{1} \cdot$ Samo Lasič $^{4} \cdot$ Peter Dahl $^{1} \cdot$ Jesper S. Hansen $^{2} \cdot$ Daniel Topgaard $^{3}$. \\ Karin Lindkvist-Petersson ${ }^{1,2}$ (I)
}

Received: 18 May 2017/ Accepted: 7 September 2017/Published online: 15 September 2017

(C) The Author(s) 2017. This article is an open access publication

\begin{abstract}
Orthodox aquaporins are transmembrane channel proteins that facilitate rapid diffusion of water, while aquaglyceroporins facilitate the diffusion of small uncharged molecules such as glycerol and arsenic trioxide. Aquaglyceroporins play important roles in human physiology, in particular for glycerol metabolism and arsenic detoxification. We have developed a unique system applying the strain of the yeast Pichia pastoris, where the endogenous aquaporins/aquaglyceroporins have been removed and human aquaglyceroporins $\mathrm{AQP} 3, \mathrm{AQP7}$, and AQP9 are recombinantly expressed enabling comparative permeability measurements between the expressed proteins. Using a newly established Nuclear Magnetic Resonance approach based on measurement of the intracellular life time of water, we propose that human aquaglyceroporins are poor facilitators of water and that the water transport efficiency is similar to that of passive diffusion across native cell membranes. This is distinctly different from glycerol and arsenic trioxide, where high glycerol transport efficiency was recorded.
\end{abstract}

Keywords Aquaporin - Aquaglyceroporin - Water transport $\cdot \mathrm{NMR} \cdot$ P. pastoris

Karin Lindkvist-Petersson

Karin.Lindkvist@med.lu.se

1 Department of Chemistry and Molecular Biology, University of Gothenburg, Box 462, 40530 Göteborg, Sweden

2 Department of Experimental Medical Science, Lund University, BMC C13, 22184 Lund, Sweden

3 Physical Chemistry, Lund University, P.O.B. 124, 22100 Lund, Sweden

4 CR Development, AB, Naturvetarvägen 14, 22362 Lund, Sweden

\section{Introduction}

Aquaporins (AQPs) are transmembrane proteins that are represented throughout all kingdoms of life, including animals and plants as well as in lower organisms such as yeast and bacteria. Their primary function is to facilitate water and glycerol transport across cell membranes (Verkman 2012). The aquaporin family is commonly divided into three sub-groups, the orthodox aquaporins (sole water facilitators), the aquaglyceroporins (that facilitate the transport of solutes such as glycerol, arsenic trioxide and urea), and the superaquaporins. There are 13 human aquaporins abbreviated AQP0-12, which are widely distributed in specific cell types in many organs and tissues (Day et al. 2014). AQP3, 7, 9, and 10 belong to the aquaglyceroporin sub-group, and in recent years, several reports suggest that the human aquaglyceroporins are essential players in human health and disease (Hara-Chikuma and Verkman 2008b; Maeda 2012). Commonly, the aquaglyceroporins are stated to have dual permeability, both for water and solutes such as glycerol (Laforenza et al. 2016). The transport specificity of the aquaglyceroporins is well-documented in the literature, frequently using either Xenopus laevis oocytes to measure the water transport rates, or artificial membranes creating liposomes with inserted proteins. In both these systems, the water transport is indirectly measured by detecting the swelling/shrinkage of the oocytes/liposomes upon an applied change in osmolality, and water transport is quantified in terms of the osmotic water permeability coefficient. Here, we apply a more direct method for studying water exchange over biological membrane by using nuclear magnetic resonance (NMR), which allows for the transport mechanisms to be studied in equilibrium conditions without applying an osmotic gradient (Eriksson et al. 2017). 
We established a unique approach to measure transport of water, glycerol, and arsenic trioxide [As(III)] in a native cell membrane using the yeast Pichia pastoris. In this approach, human AQP3, AQP7, and AQP9 are highly expressed in an AQP deletion strain of P. pastoris, where both endogenous aquaporins (Aqy1 and Agp1) have been removed by standard cloning strategies. Spheroplasts of these strains have AQP-containing lipid bilayers without requiring elaborate protein purification schemes or need of exchanging detergent micelle-stabilized membrane proteins into the lipid bilayers, necessary in liposome-based approaches. We applied the diffusion NMR method that enables quantification of the rate of water exchange between intracellular and extracellular compartments on a millisecond time-scale (Eriksson et al. 2017). Interestingly, in our system, when human aquaglyceroporins are in their native environment (lipid bilayer) and the water transport rate is measured by the non-invasive NMR method, the water transport capability of the human aquaglyceroporins are not significantly different from the passive water diffusion across the plasma membrane of cells without aquaglyceroporins expressed. Still, all aquaglyceroporins investigated significantly facilitate the transport of glycerol and As(III) in the same system. Thus, we suggest that human aquaglyceroporins are poor water facilitators compared to orthodox aquaporins, and that their main function in the body is to facilitate transport of other solutes such as glycerol and arsenic trioxide.

\section{Materials and Methods}

\section{Yeast Strains}

Pichia pastoris strains used in this study derives from the X33 strain (commercially available from Thermo Fisher, Life Technologies, USA). The protein encoded by gene PAS_chr4_0784 in P. pastoris GS115 was identified with $35 \%$ sequence identity using Fps1 as query in a BLASTp. This protein was named Aquaglyceroporin 1 (Agp1). A double-deletion strain $P$. pastoris GS115 aqy1 $:: H I S 4$ agp1 $\triangle:: N a t M X$ was generated by inserting a deletion cassette by homologue recombination into $P$. pastoris GS115 aqy1 $4:: H I S 4$ (Fischer et al. 2009). The double-cloning approach used for deleting AGPl was previously described by Ternes and co-workers (Ternes et al. 2011). A $\sim 670$ bp fragment upstream of $A G P 1$-containing restriction sites for $\mathrm{XbaI}$ and NotI and $\mathrm{a} \sim 800 \mathrm{bp}$ downstream fragment reaching into the AGPl gene containing NotI and XbaI restriction sites were amplified from genomic DNA by PCR (primer $1+2$ and $3+4$, respectively, Table 1 ). Both fragments were simultaneously ligated into the NotI site of a pPICZ $\mathrm{B}$ vector. The antibiotic resistance gene
Table 1 Primers used for cloning. Nucleic acid sequences for primers used for creating the AGP1 deletion in P. pastoris GS115 aqy1 $\triangle$ strain

\begin{tabular}{ll}
\hline Primer\# & Primer sequence $\left(5^{\prime}-3^{\prime}-\right.$ direction $)$ \\
\hline 1 & CAGTGAATTCGGAAGGTCAATCTACTACACGTG \\
2 & GACTGCGGCCGCGCAACTTTCACCAGCTTAGC \\
3 & CATAGCGGCCGCTTGTGGCTTCTGCGTTCC \\
4 & GGTGGCAGTTCTAGATCGAGG \\
5 & AAGGTTTCAGGACCTGTTGCT \\
6 & TATTCTCGTCTCCTATTGGCG \\
7 & CACAAATCCAAGAGACTGAAGAC \\
8 & CGATAGCTTGAATGTAAGTACCG \\
9 & AGCGCTTGTTCTTGAAGAGT \\
10 & ACTCTTCAAGAACAAGCGCT \\
11 & CGAGTCTTCAGTCTCTTGGAT \\
\hline
\end{tabular}

nourseothricin was cut out from the pSLnat plasmid (Ternes et al. 2011) and inserted into the NotI site of pPICZB plasmid flanked by upstream and downstream regions to the AGPl gene. The construct was confirmed by sequencing. The deletion cassette was amplified by PCR (primer 5 and 6, Table 1) and transformed into P. pastoris GS115 aqy1 $1:: H I S 4$ (Cregg 2007). Transformed cells were plated on YPD-agar plates including $1 \% \mathrm{w} / \mathrm{v}$ yeast extract, $2 \% \mathrm{w} / \mathrm{v}$ peptone and $2 \% \mathrm{w} / \mathrm{v}$ agar, $2 \% \mathrm{w} / \mathrm{v}$ glucose, and grown for 2 days at room temperature before they were replica plated onto YPDS-agar plates (YPD-agar plates $+1 \mathrm{M}$ sorbitol) + Nourseothricin (clonNAT) $(15 \mu \mathrm{g} /$ ml) (GoldBio, USA). Plates were incubated at $30{ }^{\circ} \mathrm{C}$ for 3-4 days. Transformants were re-streaked for single colonies on YPD-agar plates + clonNAT $(15 \mu \mathrm{g} / \mathrm{ml})$. Successful deletion of the AGP1 gene was verified by genomic PCR using 5 different primer pairs (primer $1+10$, $1+11,1+4,7+8$, and $9+4$, Table 1$)$.

\section{Protein Expression and Western Blot Analysis}

The human aquaporins AQP1, AQP3, AQP7, and AQP9 with a $\mathrm{His}_{6}$ purification tag were cloned into pPICZB vector using restriction enzyme EcoRI and XbaI. To identify clones that express the protein of interest, AQP transformants were spotted on YPD-agar plates supplemented with increasing zeocin concentrations $(0,0.5,1.0$, and $2.0 \mathrm{mg} / \mathrm{ml}$ ) (Thermo Fisher, Life Technologies, USA). The five most zeocin-resistant clones of each AQP construct were further analyzed for expression levels. Cells were pre-grown in buffered glycerol complex medium (BMGY: $1 \% w / v$ yeast extract, $2 \% w / v$ peptone, $100 \mathrm{mM}$ potassium phosphate $\mathrm{pH} 6.0,1.34 \% \mathrm{w} / \mathrm{v}$ yeast nitrogen base, $0.4 \mathrm{mg} / \mathrm{L}$ biotin, $1 \% v / v$ glycerol) for $24 \mathrm{~h}$, and protein production was induced with methanol to a final 
concentration of $0.25 \% v / v$ for $6 \mathrm{~h}$ (for AQP3, AQP7, AQP9) or $1 \mathrm{~h}$ (for AQP1). Cells were harvested at $2000 \times g$ for $5 \mathrm{~min}$ and washed in $20 \mathrm{mM}$ HEPES $\mathrm{pH} 7.5$. Plasma membranes were purified using the protocol of Panaretou and Piper (2006). Approximately, 300- $\mu$ l cell pellet was harvested and re-suspended in an equal volume of breaking buffer $(20 \mathrm{mM}$ Tris- $\mathrm{HCl} \mathrm{pH} 7.5,0.4 \mathrm{M}$ sucrose, $4 \mathrm{mM}$ EDTA, $2 \mathrm{mM}$ DTT) containing protease inhibitor (Roche Diagnostics) and glass beads. Cells were broken by Fast Prep (MP Biomedical, USA), cell debris was removed at $600 \times g$ for $10 \mathrm{~min}$ at $4{ }^{\circ} \mathrm{C}$, and the total membrane was collected by centrifugation at $21,000 \times g$ for $30 \mathrm{~min}$ at $4{ }^{\circ} \mathrm{C}$. Total membranes were re-suspended in membrane resuspension buffer $(10 \mathrm{mM}$ Tris- $\mathrm{HCl} \mathrm{pH} 7.5$, $2 \mathrm{mM}$ EDTA). A sucrose gradient containing equal volumes of $2.25,1.65$, and $1.1 \mathrm{M}$ sucrose in $10 \mathrm{mM}$ Tris- $\mathrm{HCl}$ $\mathrm{pH} 7.5$ and $2 \mathrm{mM}$ EDTA was overlaid with the total membrane sample and spun overnight in a SW Ti60 centrifuge at $40,000 \mathrm{rpm}$ at $4{ }^{\circ} \mathrm{C}$. The plasma membrane fraction was removed from the 2.25/1.65 $\mathrm{M}$ interphase and diluted five times into membrane resuspension buffer and spun for $30 \mathrm{~min}$ at $20,000 \times g$ at $4{ }^{\circ} \mathrm{C}$. Pellet was re-suspended in plasma membrane resuspension buffer $(20 \mathrm{mM}$ Tris-HCl pH 7.5, $150 \mathrm{mM} \mathrm{NaCl}, 10 \% v / v$ glycerol) with protease inhibitor. Purified plasma membrane samples were resolved on NuPAGE 4-12\% Bis-Tris (Thermo Fisher, Life Technologies, USA) gels and blotted onto nitrocellulose membranes (Hoefer Inc, USA). Membranes were blocked with fish gelatin blocking buffer $(10 \mathrm{mM}$ Tris- $\mathrm{HCl} \mathrm{pH} 7.5,150 \mathrm{mM} \mathrm{NaCl}, 2 \% w / v$ Fish Gelatin, $1 \% \mathrm{w} / \mathrm{v}$ Ovalbumin). Antibodies were incubated with blocked membrane in 1:1 blocking buffer and $20 \mathrm{mM}$ Tris-HCl pH 7.5, $100 \mathrm{mM} \mathrm{NaCl}$ with $0.1 \% v / v$ Tween-20 (TBS-T). The plasma membrane marker, Pma1, was detected using rabbit-anti-Pmal antibody (Santa Cruz Biotechnology, USA; 1:1,000, \#sc-33735) at $4{ }^{\circ} \mathrm{C}$ overnight and the His-tag was detected with the mouse-anti-His antibody (Sigma-Aldrich, USA; 1:5000) for $45 \mathrm{~min}$ at room temperature. Donkey-anti-mouse $680 \mathrm{~nm}(1: 10,000)$ and donkey-anti-rabbit $800 \mathrm{~nm}(1: 10,000)$ were used as secondary antibodies, and incubated for $45 \mathrm{~min}$ at room temperature (LI-COR, USA). Fluorescent signals were detected using Odyssey FC (LI-COR, USA). The endoplasmic reticulum (ER) marker, Sec61, antibody was used at 1:1000 and incubated over night at $4{ }^{\circ} \mathrm{C}$. The signal was detected using an anti-rabbit horseradish peroxidase antibody and enhanced chemiluminescence (ECL). The signal was detected by the Gel DocTMsystem (BIO-RAD, USA).

\section{Diffusion NMR}

Cells were grown and protein production induced as described above. Cells were harvested and washed with
$20 \mathrm{mM}$ HEPES $\mathrm{pH} 7.5$, re-suspended in a $1: 1 v / v$ solution with buffer, and transferred to 5-mm disposable NMR tubes and centrifuged at $1000 \times g$ for $10 \mathrm{~min}$ to achieve a pellet with high concentration of cells. The tubes were kept on ice until measurement. The NMR diffusion measure-

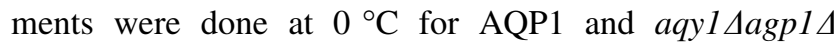
induced for $1 \mathrm{~h}$ and at $20^{\circ} \mathrm{C}$ for strain $\mathrm{AQP} 3,7$, and 9 and aqyl $\Delta a g p 1 \Delta$ induced for $6 \mathrm{~h}$. According to Eriksson et al., the temperatures and induction times were chosen so that the same set of mixing times $\left(t_{\mathrm{m}}\right)$ allowed a precise quantification of exchange for all strains, aqyl $\Delta a g p 1 \Delta$,

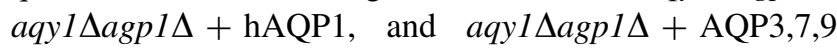
(Eriksson et al. 2017). Measurements were performed on a Bruker $200 \mathrm{MHz}$ Avance-II spectrometer with a DIF-25 gradient probe capable of $z$-gradients up to $9.6 \mathrm{~T} / \mathrm{m}$. The NMR method used is described in Eriksson et al. (Eriksson et al. 2017) and identical experimental parameters were used here. The method combines a filter exchange spectroscopy (FEXSY) pulse sequence with a pulsed-gradient spin-echo (PGSE). The FEXSY experiment consists of two diffusion-encoding blocks separated by a mixing time, $t_{\mathrm{m}}$, and is specifically sensitive to exchange. The PGSE pulse sequence was applied with varying echo time, $t_{\mathrm{E}}$, to correlate the intra- and extracellular diffusion coefficients and $T_{2}$-relaxation times and was used to compensate for differences in $T_{2}$-relaxation between the intra- and extracellular water. The intra- to extracellular exchange rate was obtained by a constrained global fitting of both datasets.

\section{Stopped Flow Measurements}

Cells were isolated at $2000 \times g$ for $5 \mathrm{~min}$ and washed three times in $20 \mathrm{mM}$ HEPES pH 7.5 and $1.2 \mathrm{M}$ Sorbitol, and resuspended to a final absorbance at $\mathrm{A}_{600}$ of 3 . In the Stopped Flow Machine (SFM) procedure, cells were subjected to a hyperosmotic shock (20 mM HEPES pH 7.5 and $1.8 \mathrm{M}$ Sorbitol) with a mixing rate of $7 \mathrm{ml} / \mathrm{s}$ in a ratio of $1: 1$ to a final volume of $148 \mu$ l. The cell response (shrinkage) was monitored as increased light-scattering intensity at $435 \mathrm{~nm}$ at an angle of $90^{\circ}$ using a SFM-20 and MOS-450 spectrometer (BioLogic).

\section{Glycerol Transport Measurement}

Washed cells were re-suspended to an approximate density of $30 \mathrm{mg} / \mathrm{ml}$, and subsequently the absorbance at $\mathrm{A}_{600}$ was measured and precisely adjusted for all strains. Carbonyl Cyanide m-Chlorophenylhydrazone (CCCP) was added to a final concentration of $100 \mu \mathrm{M}$ to the cell suspension to inhibit uptake of glycerol by active transporters, and equilibrated with the cells at $30{ }^{\circ} \mathrm{C}$ for $10 \mathrm{~min}$ prior to each measurement. Uptake studies were initiated by the addition of a glycerol mix $\left(300 \mathrm{mM}\right.$ glycerol and $40 \mu \mathrm{M}\left[{ }^{14} \mathrm{C}\right]$ 

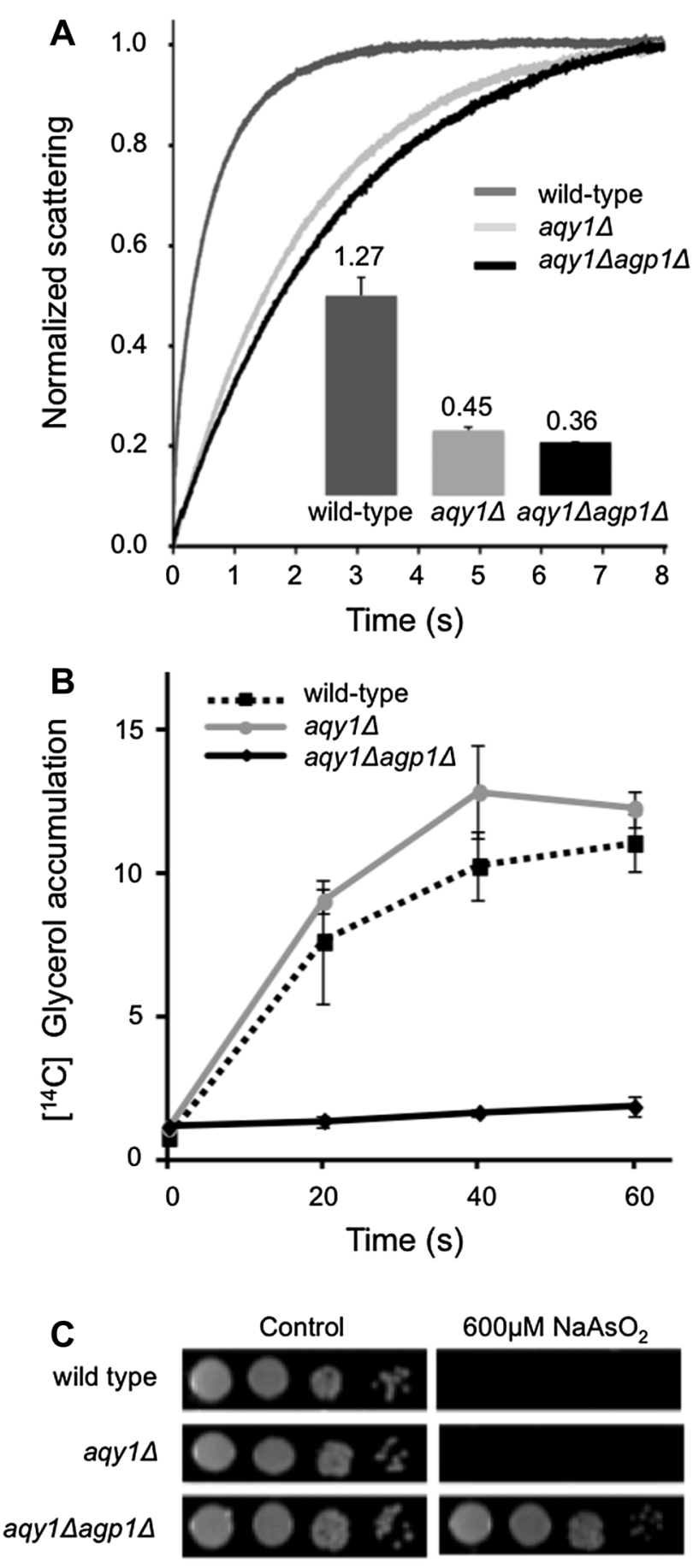

glycerol (142.7 $\mathrm{mCi} / \mathrm{mmol}$; Perkin Elmer)). The reaction was stopped by transferring the whole mixture to a prefilled funnel with $5 \mathrm{ml}$ pre-chilled $20 \mathrm{mM}$ HEPES, $\mathrm{pH} 7.5$, $500 \mathrm{mM}$ glycerol. Cells were then washed and disintegration per minutes (dpm) was recorded as previously described (Tamas et al. 1999). The measured signal in dpm was converted to nmol glycerol per OD unit and plotted over time. For measurement carried out at pH 6.0, $20 \mathrm{mM}$ MES pH 6.0 was used instead of $20 \mathrm{mM}$ HEPES pH 7.5.
4Fig. 1 A novel yeast strain of $P$. pastoris that is impermeable for water, glycerol, and arsenic trioxide. Comparison of solute permeation in different strains of $P$. pastoris. a Cells were subjected to a hyperosmotic shock causing outward movement of water. Normalized Light Scatter Intensity (NLSI) was plotted over time (s). The rate constant, $k$, was obtained by curve fitting using the equation: $y=$ $A_{1} \cdot e^{-k_{1} \cdot t}+A_{2}$ ( $A_{1}$ Amplitude, $k$ rate constant, $t$ time, $A_{2}=$ offset $)$, and are shown as bars (inset), values are mean of duplicates, and error bars denote SD. b Glycerol accumulation per OD unit measured as a function of time after addition of $\left[{ }^{14} \mathrm{C}\right]$ glycerol. Values shown are mean normalized to the mean 0 -value in each experiment and error bars denote standard deviation (SD). c Cells were spotted on Control media (BMMY, Buffered Methanol complex Media) with and without $\mathrm{NaAsO}_{2}$ in 10 times dilution series to monitor As(III) sensitivity. A representative image is shown

\section{As(III) Transport and Phenotype Assay}

For each individual measurement, $10 \mathrm{OD}$ units of washed cells were re-suspended in $20 \mathrm{mM}$ HEPES $\mathrm{pH} 7.5$ and incubated at $30{ }^{\circ} \mathrm{C}$ for 5-10 min prior measurement. Uptake studies were initiated by the addition of $\mathrm{NaAsO}_{2}$ in $1: 1(\mathrm{v} / \mathrm{v})$ ratio with a final concentration of $100 \mathrm{mM}$. The reaction was stopped by transferring the cell suspension to cold $20 \mathrm{mM}$ HEPES buffer $\mathrm{pH} 7.5$ and collected on a Whatman ${ }^{\circledR}$ glass microfiber filter Grade GF/C and washed twice in cold $20 \mathrm{mM}$ HEPES buffer. Cells were re-suspended in water and disrupted by boiling. Cell debris was sedimented at $10,000 \times g$ for $10 \mathrm{~min}$. Sodium arsenite-containing supernatants were sent to analysis by inductively coupled plasma mass spectrometry (ICP-MS). For the growth assay, cells were cultivated and protein expression was induced (as described above). Cells were diluted in $20 \mathrm{mM}$ HEPES pH 7.5 to an absorbance at $\mathrm{A}_{600}$ of 0.2 . Five microliters from 10 times dilution series were spotted on agar plates containing buffered methanol complex medium (BMMY: 1\% w/ $v$ yeast extract, $2 \% \mathrm{w} / v$ peptone, $100 \mathrm{mM}$ potassium phosphate $\mathrm{pH} 6.0,1.34 \% \mathrm{w} / \mathrm{v}$ yeast nitrogen base, $0.4 \mathrm{mg} / \mathrm{l}$ biotin, $0.5 \% \mathrm{v} / \mathrm{v}$ methanol, $2 \% \mathrm{w} / \mathrm{v}$ agar) with and without $\mathrm{NaAsO}_{2}(0$ and $600 \mu \mathrm{M})$ and incubated at $30{ }^{\circ} \mathrm{C}$ for 3 days.

\section{Statistical Analysis}

Comparisons between multiple groups were done in GraphPad Prism by one-way ANOVA followed by Tukey's multiple comparisons test and the null hypothesis was rejected at the 0.05 confidence level.

\section{Results and Discussion}

The yeast $P$. Pastoris has been successfully used for heterologous protein production and, particularly, in recent years also for overexpressing transmembrane proteins. Thus, $P$. pastoris is a suitable system for measuring the 
Fig. 2 Quantification of aquaglyceroporins levels in the plasma membrane.

a Quantitative Western blot analyses of aquaglyceroporins present in the plasma membrane. Protein content in plasma membrane fractions were analyzed by SDS-PAGE and Western blotting detecting Pma1 membrane marker and the polyhistidine-tag of the AQPs.

Note that the typical aquaporin migrates in the gel as different mono/oligomers.

b Quantification of the aquaglyceroporins present in the plasma membrane (His-signal divided by Pma1-signal), values are mean, and error bars denote SD

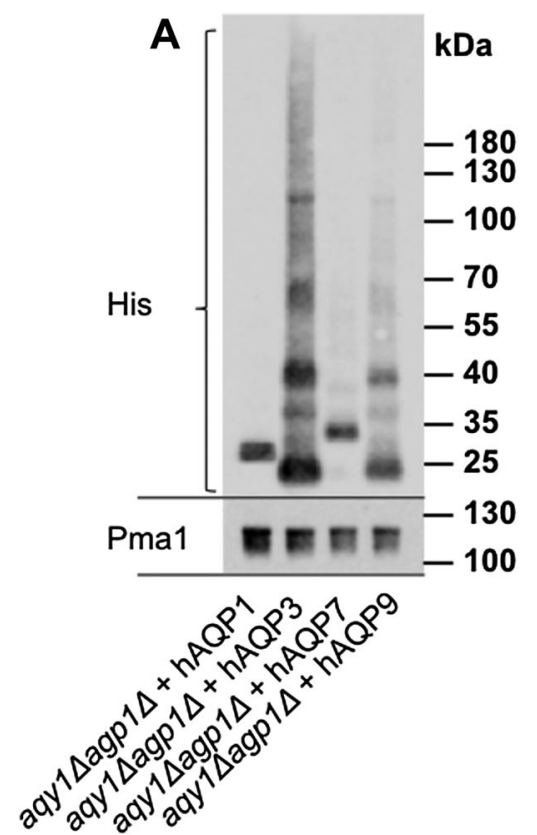

B

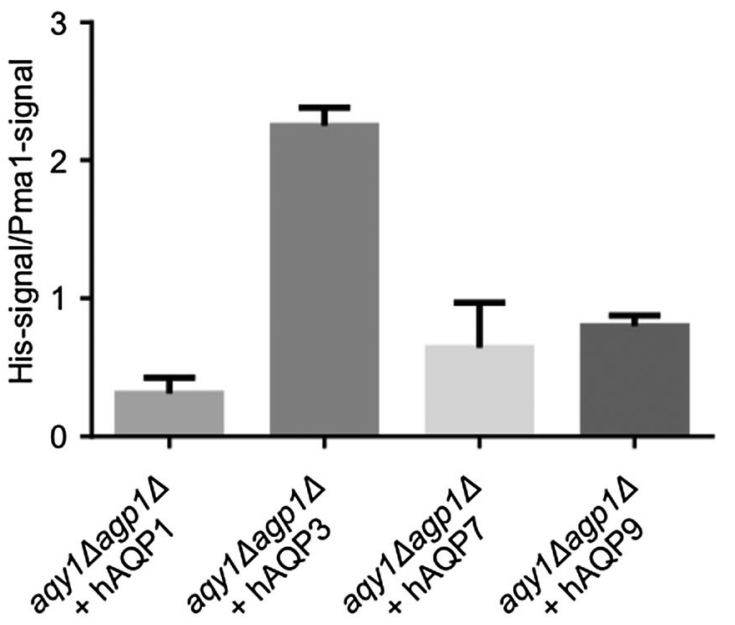

activity of human transmembrane proteins. In this study, we aim at pinpointing the detailed specificity of the human aquaglyceroporins. The genome for $P$. pastoris GS115 has been public since 2009 (De Schutter et al. 2009) and contains two aquaporin genes encoding the previously characterized Aqy1 (Fischer et al. 2009) and a second putative aquaglyceroporin, Agp1 (AquaGlyceroPorin1).

\section{A Novel System to Study Substrate Specificity of Human Aquaglyceroporins}

To enable functional studies of human aquaglyceroporins, a $P$. pastoris strain deficient in endogenous aquaporins, Aqy1 and Agp1 proteins, was generated. P. pastoris has previously been used to measure water transport rate of heterologously expressed aquaporins, however, both the endogenous aquaporins of $P$. pastoris were not removed in those studies, which may impact the results (Azad et al. 2009; Fischer et al. 2009). To confirm that the transport abilities typical for both aquaporins and aquaglyceroporins are disrupted in our strain, water, glycerol, and As(III) transport efficiency was measured in a $P$. pastoris wild-type strain (X33), single-deletion strain $P$. pastoris GS115 aqy14 (where the orthodox aquaporin is deleted) (Fischer et al. 2009) and a new double-deletion strain $P$. pastoris GS115 aqy1 LagplA (where both AQY1 and aquaglyceroporin, $A G P 1$ are deleted) (Fig. 1). The water transport rates were assayed using the standard stopped-flow light-scattering technique, where spheroplasts of the three different strains (wild type, aqy1 , aqy1 Aagp1 1 ) were subjected to a hyperosmolality solution and the concomitant change in light scattering was measured as a function of time (Fig. 1a). The wild-type strain, containing both aquaporins, had a rate constant around three times higher the values of both the aqy $1 \Delta$ strain and the aqyl $\Delta a g p l \Delta$ strain, suggesting that it has roughly three times higher water transport (Fig. 1a). Marginal differences in water transport rates are observed between the two deletion strains, which are interpreted as Agp1 is not considerably contributing to the water efflux and hence has poor water transporting capability (Fig. 1a). Glycerol uptake efficiency was measured as accumulation of radiolabeled glycerol over time (Fig. 1b). The wild type and the aqy1 $\Delta$ strain readily accumulated glycerol, while the aqy1 $\Delta$ agp $1 \Delta$ strain did not, suggesting that Agp1 is a glycerol conducting channel. To test whether arsenic trioxide, a highly toxic compound, is a potential substrate for Aqy1 and/or Agp1, cells from the three different strains were spotted on As(III)containing plates (Fig. 1c). In this assay, cells expressing efficient As(III) facilitators will not survive. Cell growth was clearly affected in both wild type and $a q y 1 \Delta$ strains, while the aqyl 1 agpl 1 strain showed tolerance towards arsenic trioxide. This suggests that both the wild-type strain and the aqy1 $\Delta$ strain take up As(III), while the aqyl 1 agpl $\Delta$ strain cannot, and hence Agp1 is likely responsible for the uptake. Taken together, by deleting $A Q Y 1$ and AGP1 in P. pastoris, we have generated a novel system where heterologous aquaporins can be expressed and their substrate specificity for water, glycerol, and As(III) can be evaluated.

\section{The Human Aquaglyceroporins are Poor Water Facilitators}

To investigate the transport specificity of the human aquaglyceroporins for water, glycerol, and As(III), hAQP1, hAQP3, hAQP7, and hAQP9 were expressed in the double- 

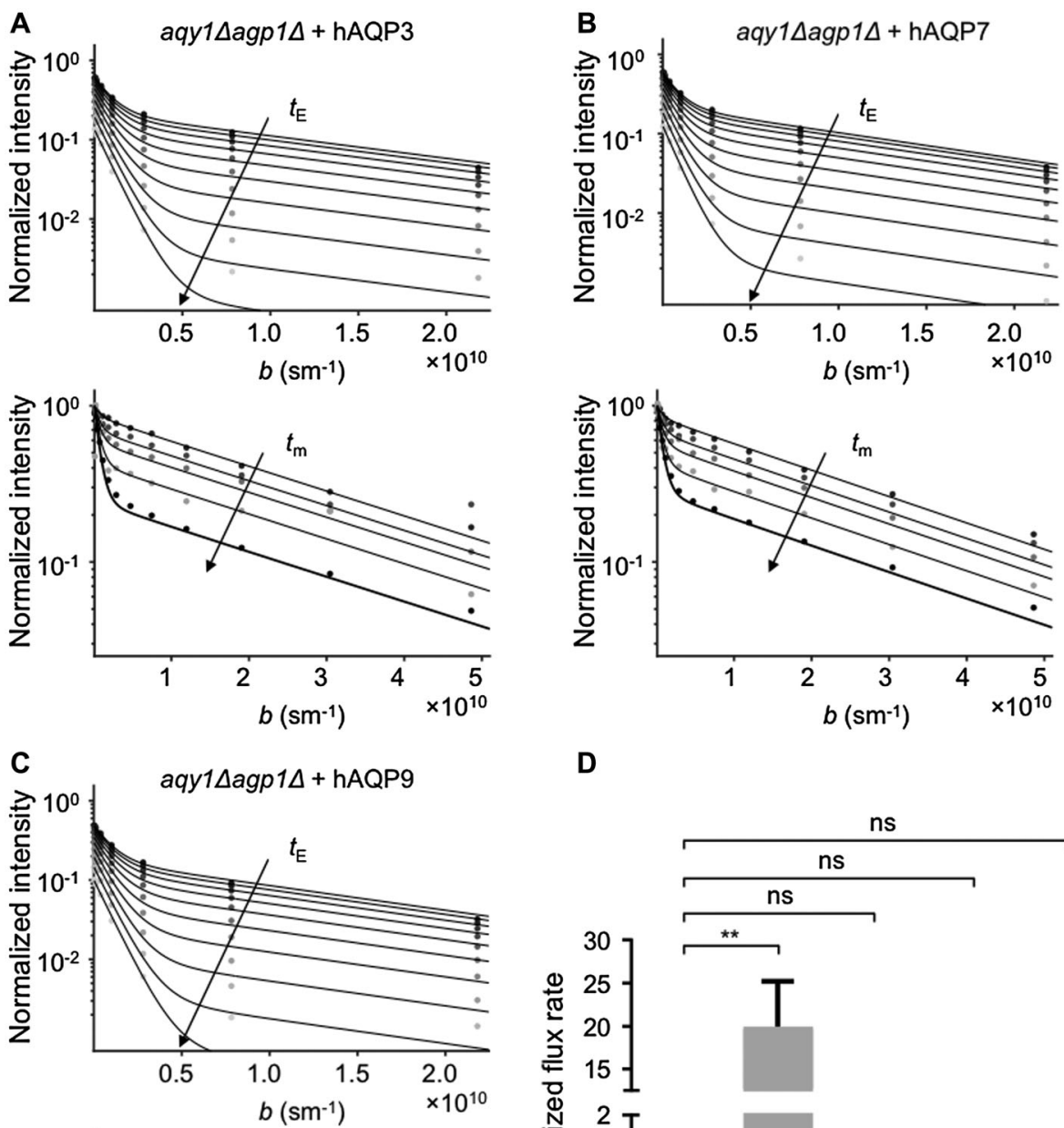

D

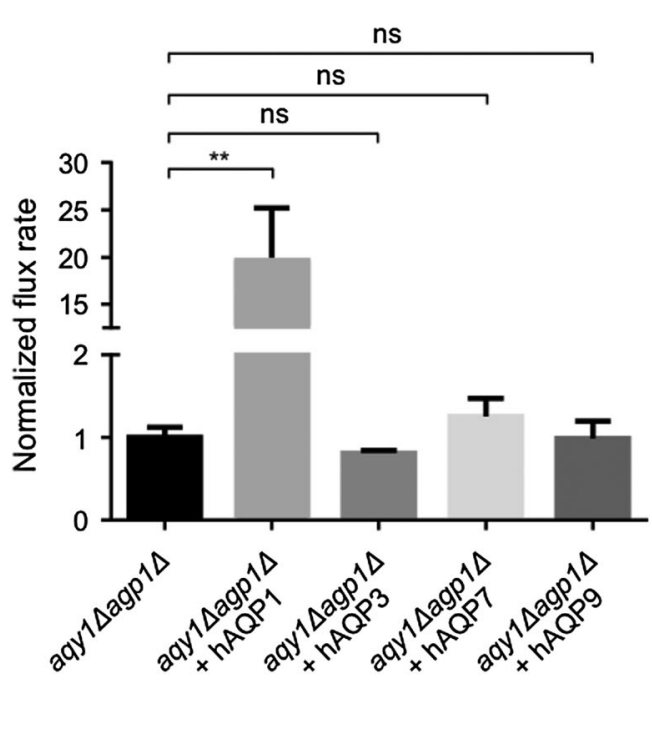

Fig. 3 Diffusion NMR method used to measure water exchange in cells. Water transport efficiency was measured for the different strains using the NMR diffusion technique. a-c Examples of results from NMR diffusion experiments showing normalized signal intensity for (a) aqy1 $\Delta a g p 1 \Delta+\mathrm{hAQP}$, (b) aqy1 4 agp1 $1+\mathrm{hAQP} 7$, and (c) aqy1 1 agp 1 $+\mathrm{hAQP} 9$ as a function of diffusion weighting $b$. The upper graph shows signal attenuation curves from the PGSE experiment for increasing echo times, $t_{\mathrm{E}}$, indicated by arrow. The lower graph shows signal attenuation curves from the FEXSY experiment where arrow indicates increasing mixing times, $t_{\mathrm{m}}$.

(d) Statistical significance was established by one-way ANOVA $(F(4,10)=11.27, p=0,001)$, and Tukey's multiple comparisons test rendered statistical significant differences $(p<0.01)$ exclusively between aqy1 $\triangle$ agp1 $\Delta+\mathrm{hAQP} 1$ and the other strains (aqy1 $\Delta a g p 1 \Delta$,

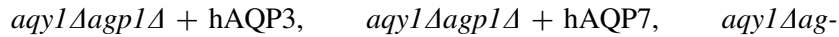
pl $1+\mathrm{hAQP9}$ ), but non-significant differences in all other comparisons. The aqy1 4 agpl $1+\mathrm{hAQP} 1$ strain was analyzed at $0{ }^{\circ} \mathrm{C}$ together with aqy1 $\operatorname{agp} 1 \Delta$ control, while the other strains were analyzed at $20{ }^{\circ} \mathrm{C}$. Values are mean values normalized to aqyl $\Delta a g$ $p 1 \Delta$ in each experiment ( $\left.\mathrm{ki} / \mathrm{ki}_{\text {aqy } 1 \Delta a g p 1 \Delta}\right)$ and error bars denote SEM

deletion strain of $P$. pastoris (aqyl Aagp1 $\Delta$ ) (Fig. 2a). The levels of protein expression in the plasma membrane were quantified using Western blotting of plasma membrane fractions, detecting the histidine purification tag fused to the respective aquaporin (His) and a plasma membrane

marker (Pma1), to relate aquaporin levels to the plasma membrane purity of the fraction samples (Fig. 2a, b). Previously, the $X$. laevis oocyte expression system has been widely used to investigate the permeability of water for human AQP3, AQP7, and AQP9, and all of them facilitated 


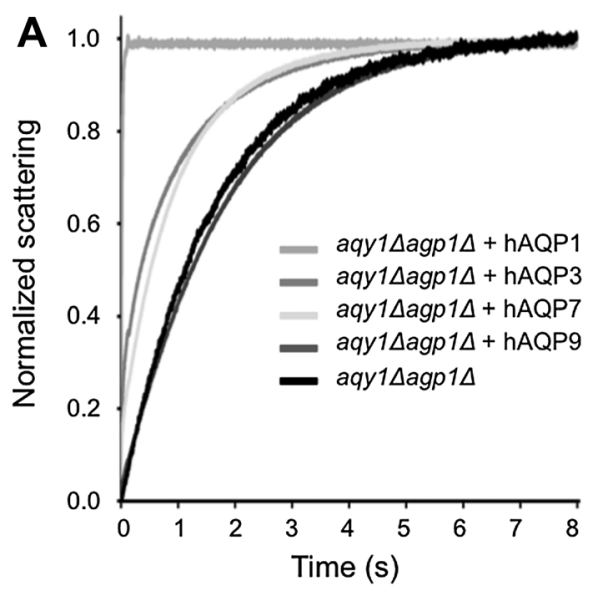

Fig. 4 Applying SFM to measure cell shrinkage as indicative for water efflux. a Cells were subjected to a hyperosmotic shock causing outward movement of water. Normalized Light Scatter Intensity (NLSI) was plotted over time (s). b Water exchange rate constant

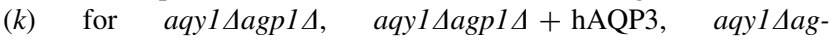
pl $1+\mathrm{hAQP7}$, aqy1 1 agp1 $1+\mathrm{hAQP9}$, values are mean of

the transport of water in that system (Boury-Jamot et al. 2006; Ishibashi et al. 1998; Kondo et al. 2002). Along those lines, human AQP3 has also been shown to facilitate the transport of water in liposomal model membranes (MullerLucks et al. 2013). In these previous studies, water transport was measured indirectly as the water transport activity is estimated by the volume change of the oocyte/liposome after a rapid change in osmolality of the surrounding medium. We have applied an NMR diffusion technique to measure the actual rate of water exchange between intracellular and extracellular space across a native cell membrane. The intracellular lifetime of water molecules was determined by quantifying the mean squared displacement of water molecules as a function of diffusion time on a millisecond time-scale (Eriksson et al. 2017). In contrast to previous reports, our NMR results, where the intra- to extracellular exchange rate was obtained by a constrained global fitting, show that aquaglyceroporins are poor water facilitators, as compared to the orthodox aquaporin AQP1 (Fig. 3). Their transport activity was measured to be in the same range as passive water diffusion over the membrane (Fig. 3d). To compare the results from the novel NMR method with standard indirect analysis of water permeability using the stopped-flow light-scattering technique, spheroplasts of the double-deletion strain expressing human AQP1, AQP3, AQP7, and AQP9 were exposed to a hyperosmolal solution and the change in light scattering was measured. Interestingly, spheroplasts expressing the human aquaglyceroporins still exhibit poor water transport as evidenced by rate constants of $0.5-1.0 \mathrm{~s}^{-1}$, which are comparable to the background strain (Fig. 4). On the other hand, spheroplasts expressing the orthodox AQP1 has a strikingly higher transport rate (Fig. 4a), previously

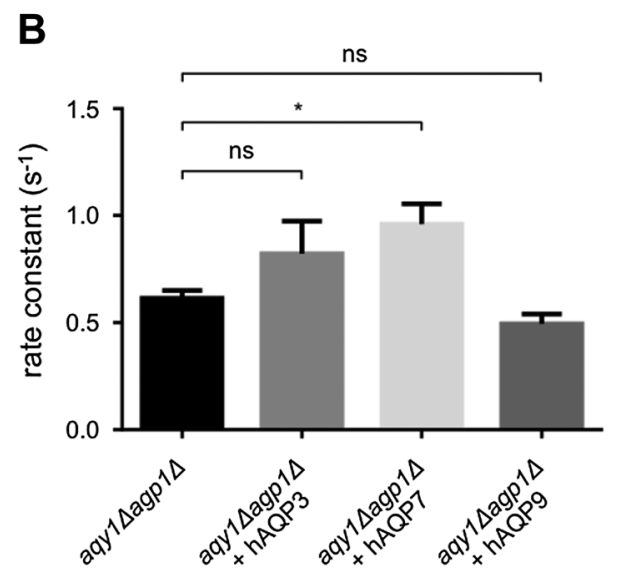

triplicates, and error bars denote SEM. Statistical significance was investigated with one-way $\operatorname{ANOVA}(F(3,8)=14.38, p<0.01)$, and Tukey's multiple comparisons test rendered statistical significant

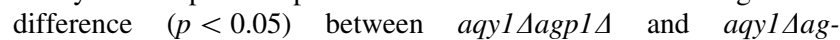
$p 1 \Delta+\mathrm{hAQP} 7$ and non-significant differences comparing aqy1 $\mathrm{a} a \mathrm{~g}$ p1 $\Delta$ with aqy1 4 agpl $\Delta+\mathrm{hAQP} 3$ and aqy1 1 agp $1 \Delta+\mathrm{hAQP} 9$

estimated to be about ten times faster than passive diffusion (Fischer et al. 2009). The discrepancy between our results and the results from previous reports using $X$. laevis oocytes and model membranes, may be explained by the fact that in our system the aquaglyceroporins are in native cell membranes. In fact, water transport has previously been investigated in native membranes for the three investigated aquaglyceroporins. For instance, after deletion of the $A Q P 3$ gene in human erythrocytes, no reduction in water transport was detected (Roudier et al. 2002), similarly deletion of the $A Q P 7$ gene in kidney brush-border membranes made into vesicles, showed osmotic water permeability in the same range as published for AQP7 expressed in Xenopus oocytes $\left(P_{\mathrm{f}} \sim 0.015 \mathrm{~cm} / \mathrm{sec}\right)($ Geyer et al. 2013; Kondo et al. 2002; Sohara et al. 2005). Finally, after deletion of $A Q P 9$ gene in erythrocytes, there was no detectable effect of water permeability (Liu et al. 2007) and along those lines, rat $\mathrm{AQP} 3$ and rat $\mathrm{AQP} 9$ and another aquaglyceroporin from Plasmodium falciparum, PfAQP, were evaluated for water activity when expressed in the native lipid bilayer in the yeast Saccharomyces cerevisiae, and showed to have comparable water transport to that of passive diffusion (Hedfalk et al. 2008; Pettersson et al. 2006). Taken together, these data strongly support the notion that human aquaglyceroporins have very low water transport activity when expressed in native membranes; comparable to the passive diffusion of water over the plasma membrane (Figs. 3, 4). However, there are reports from rodent knock-out models and cell lines showing deletion of at least mouse AQP3 could affect the water transport activity in mouse cells, indicating that there may be differences between species (Hara-Chikuma and Verkman 2008a). 

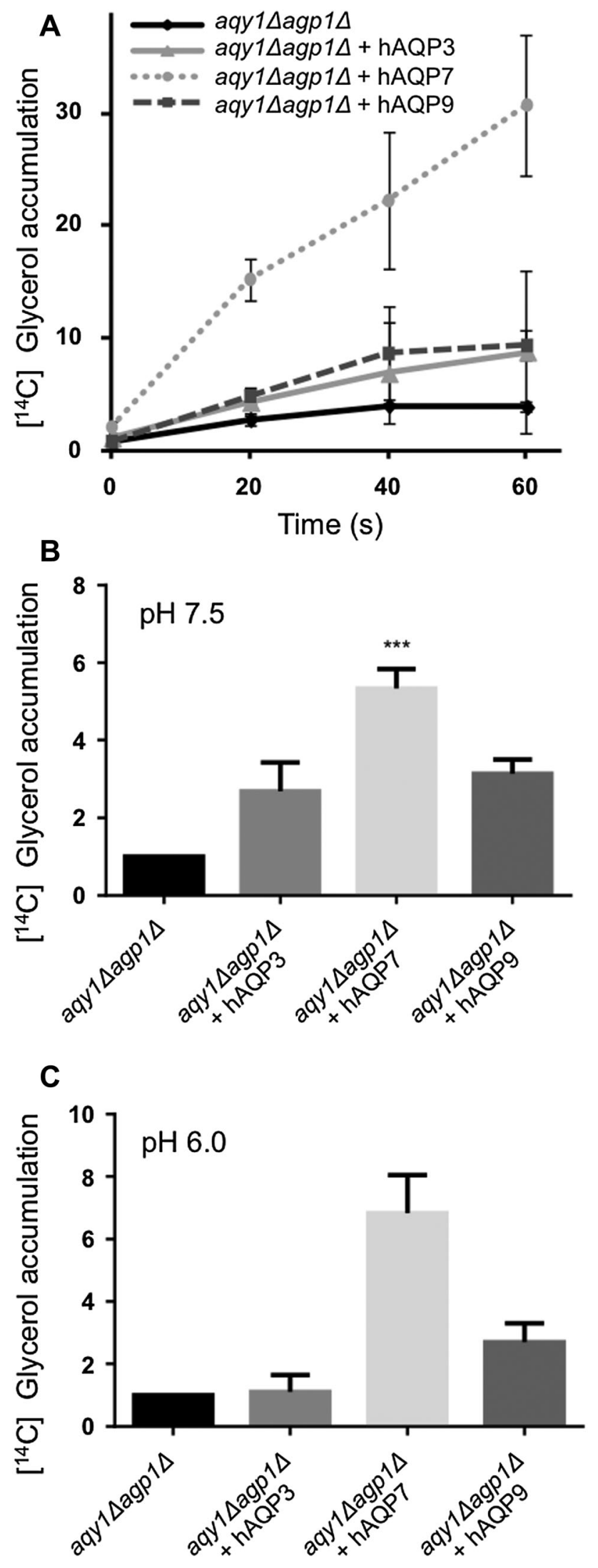

4Fig. 5 The human aquaglyceroporin are efficient glycerol facilitators. Human aquaglyceroporins overexpressed in aqyl $\Delta a g p 1 \Delta$ double-deletion strain were tested for their glycerol transport properties at two different $\mathrm{pHs}$ by supplementing a mix of glycerol and ${ }^{14} \mathrm{C}$ glycerol. a At pH 7.5, cells were exposed to glycerol and the accumulation plotted at different time points. The relative glycerol uptake per OD unit is plotted (normalized to the mean 0-value in each experiment, and error bars shows SD). b Relative glycerol uptake per OD unit at $30 \mathrm{~s}(\mathrm{pH} 7.5)$, values are mean of triplicates normalized to

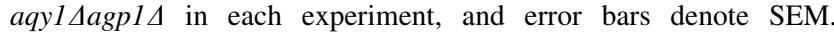
c Relative glycerol uptake per OD unit at $30 \mathrm{~s} \mathrm{(pH} \mathrm{6.0),} \mathrm{values} \mathrm{are}$ mean of duplicates normalized to aqyl $\Delta a g p 1 \Delta$ in each experiment, and error bars denote SD

\section{The Human Aquaglyceroporins Facilitate the Transport of Glycerol}

The main substrate for aquaglyceroporins is known to be glycerol and the three human aquaglyceroporins AQP3, AQP7, and AQP9 have all previously been shown to transport glycerol in $X$. laevis oocytes (Chauvigne et al. 2011; Kondo et al. 2002; Ohgusu et al. 2008). In our system, we detected water transport rates for the human aquaglyceroporins at levels comparable to that of passive diffusion. To confirm that the $P$. pastoris system does not negatively influence the transport capability of the proteins in general, glycerol accumulation was measured for the different human aquaglyceroporins. At $\mathrm{pH} 7.5$, glycerol accumulation was observed for all strains expressing the aquaglyceroporins although with somewhat varying efficiency (Fig. 5a, b). AQP7 was the most pronounced glycerol facilitator, whereas cells expressing AQP3 and AQP9 only accumulated approximately half the glycerol amount compared to AQP7 (Fig. 5b). At pH 6.0, AQP3 lost its glycerol transport ability (Fig. 5c), which is consistent with earlier observations (Zeuthen and Klaerke 1999), and also confirm proper functionality of the human AQPs in the yeast system. The transport efficiency of AQP7 and AQP9 was as expected, not affected by the change in $\mathrm{pH}$ (Fig. 5c). Thus, all the aquaglyceroporins are functional glycerol channels when expressed in the double-deletion $P$. pastoris strain, and hence dysfunctional proteins cannot explain the low water uptake.

\section{AQP7 and AQP9 are Potent Arsenic Trioxide Facilitators}

The aquaglyceroporins are also known to facilitate the transport of arsenic trioxide, As(III), and As(III) accumulation has previously been measured for the human aquaglyceroporins using X. laevis oocytes (Liu et al. 2004) and in another yeast, Saccharomyces cerevisiae (Liu et al. 2002). In both systems, human AQP7 and AQP9 were concluded to be efficient As(III) facilitators, while AQP3 
Fig. 6 Human AQP7 and AQP9 are efficient As(III) facilitators. a Cells are spotted in 10-time dilution series on Control (BMMY, Buffered Methanol complex Media) and with $\mathrm{NaAsO}_{2}$ supplemented. b Accumulated As(III) concentrations at $t=300 \mathrm{~s}$ the presence of $100 \mathrm{mM} \mathrm{NaAsO}$ determined using ICP-MS.

Values are mean of triplicates normalized to aqy1 $\Delta a g p 1 \Delta$ in each experiment, and error bars denote SEM
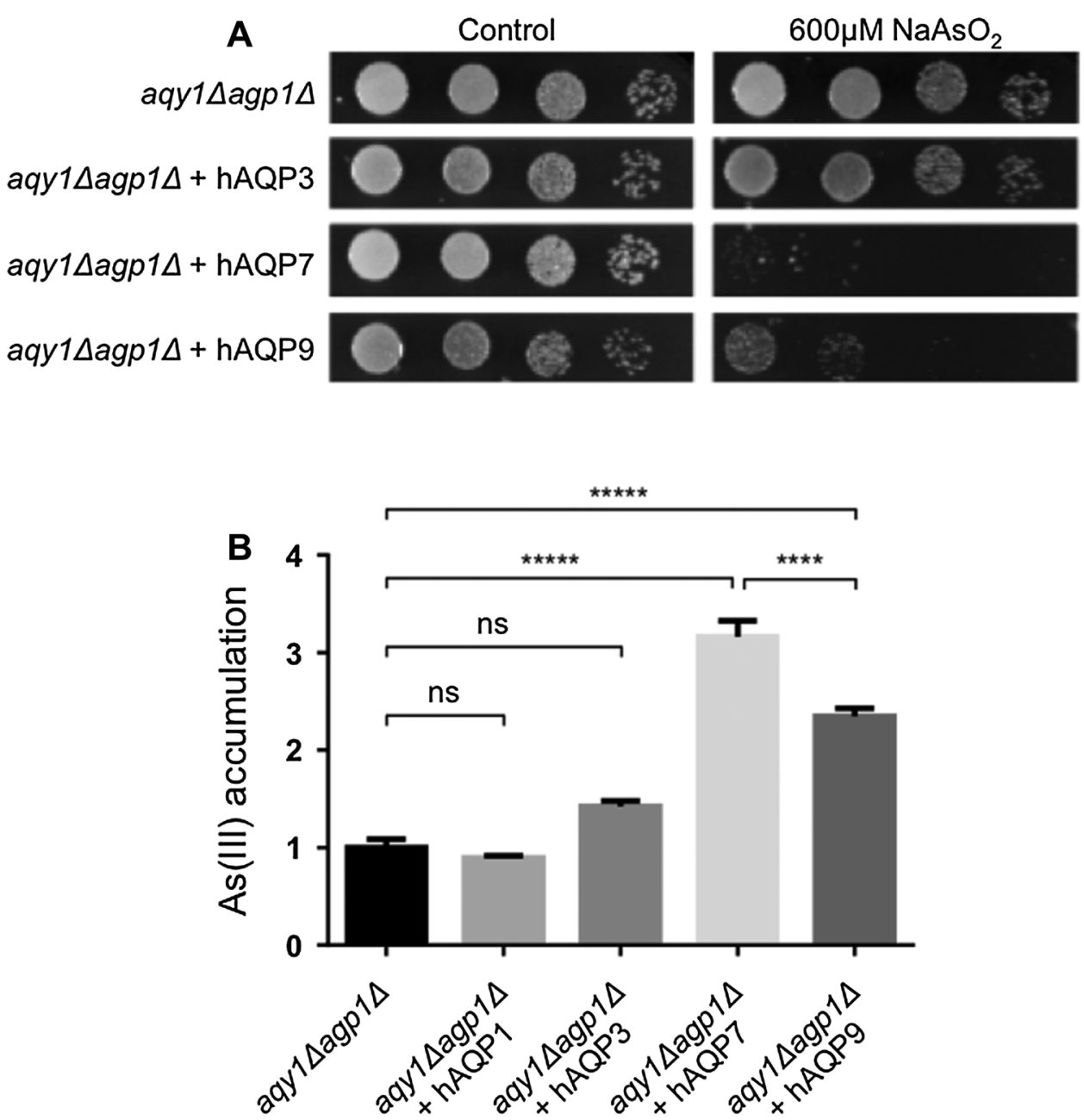

was shown to be a poor As(III) facilitator in $X$. leavis oocytes. In the $P$. pastoris double-deletion strain, cells expressing AQP7 and AQP9 had difficulties to survive at high concentrations of As(III), indicating that AQP7 and AQP9 are As(III) facilitators (Fig. 6a), which is in accordance with previous findings (Liu et al. 2002, 2004). Cells expressing AQP3 were, however, unaffected, suggesting that AQP3 is a poor facilitator of As(III) (Fig. 6a), which is also consistent with previously data from the $X$. laevis oocyte system (Liu et al. 2004). To investigate the uptake rates of $\mathrm{As}(\mathrm{III})$, the intracellular As(III) levels were measured using inductively coupled plasma mass spectrometry (ICP-MS). In agreement with the growth assays, cells expressing human AQP7 and AQP9 accumulated As(III), while cells expressing AQP3 did not significantly accumulate As(III) compared to the double AQP deletion strain (Fig. 5b). These results show that AQP7 and AQP9 are much more potent As(III) facilitators compared to AQP3, which correlates well with the growth phenotypes (Fig. 6a). AQP1 was included as a negative control and did not facilitate transport of $\mathrm{As}$ (III) (Fig. 6b). In addition,
AQP7 was shown to exhibit significantly higher arsenic trioxide transport efficiency than AQP9 (Fig. 6b), which has recently also been confirmed in the $X$. laevis oocyte system (McDermott et al. 2010). This contradicts data presented by Liu and co-workers, where AQP9 was four times as efficient in As(III) transport compared to AQP7 (Liu et al. 2004). However, Liu and co-workers used a truncated variant of AQP7, which most likely explains why the authors detected lower As(III) transport efficiency for AQP7 (Liu et al. 2004). The finding that AQP7 is a very efficient As(III) transporter may have implications for human physiology and disease. In particular, arsenic trioxide is efficacious compound in treatment of acute promyelocytic leukemia (APL) and the expression levels of AQP9 in primary leukemia samples have been suggested to be a useful predictor for the efficacy of arsenic therapy (Leung et al. 2007). Since human AQP7 seems to be an even more efficient As(III) facilitator, the expression levels of AQP7 should also be considered in accurately evaluating the potential therapeutic benefits of arsenic trioxide in treating APL. 


\section{Conclusions}

Human aquaglyceroporins are commonly referred to as channels facilitating the transport of glycerol in addition to water. Available methods for estimating the permeability of water through AQP channels are mainly restricted to non-polarizable cells (oocytes) or model membranes. This study uses a novel yeast-based system combined with a newly developed NMR method, and suggests that the main role of aquaglyceroporins is not related to water movement, but rather transport of glycerol and arsenic trioxide across the plasma membrane. Human aquaglyceroporins are likely not able to exclude water entirely, but the transport rates are not significantly different from passive diffusion of water across the plasma membrane, and hence likely of limited physiological relevance.

Acknowledgements We would like to express our gratitude to Dr. Örjan Hansson at University of Gothenburg for his help with stopped flow analysis, Dr. Christoph Basse Karlsruhe at Institute of Technology for providing us with pSLNAT and Jennifer Carbrey, Duke University Medical Center for providing us with human AQP9 gene. Sec61 antibody was kindly provided by Prof. Thomas Sommer, MaxDelbrück-Center for Molecular Medicine/Humboldt-University of Berlin. This project was funded by the Swedish Research Council (2011-2891 and 2016-6214), the Cancer Foundation (2010/1171 and 2014/575), the Novo Nordisk Foundation (9807), AFA Försäkring, the Olle Engkvist Byggmästsre, VINNOVA and Crafoord Foundation (20160579), all received by Karin Lindkvist-Petersson.

\section{Compliance with Ethical Standards}

Conflict of interest The authors declare that they have no conflict of interest.

Ethical Statement This article does not contain any studies with human participants or animals performed by any of the authors.

Open Access This article is distributed under the terms of the Creative Commons Attribution 4.0 International License (http://crea tivecommons.org/licenses/by/4.0/), which permits unrestricted use, distribution, and reproduction in any medium, provided you give appropriate credit to the original author(s) and the source, provide a link to the Creative Commons license, and indicate if changes were made.

\section{References}

Azad AK, Sawa Y, Ishikawa T, Shibata H (2009) Heterologous expression of tulip petal plasma membrane aquaporins in Pichia pastoris for water channel analysis. Appl Environ Microbiol 75:2792-2797. doi:10.1128/AEM.02335-08

Boury-Jamot M, Sougrat R, Tailhardat M, Le Varlet B, Bonte F, Dumas M, Verbavatz JM (2006) Expression and function of aquaporins in human skin: is aquaporin-3 just a glycerol transporter? Biochem Biophys Acta 1758:1034-1042. doi:10. 1016/j.bbamem.2006.06.013

Chauvigne F, Lubzens E, Cerda J (2011) Design and characterization of genetically engineered zebrafish aquaporin-3 mutants highly permeable to the cryoprotectant ethylene glycol. BMC Biotechnol 11:34. doi:10.1186/1472-6750-11-34

Cregg JM (2007) DNA-mediated transformation methods in molecular biology 389:27-42. doi:10.1007/978-1-59745-456-8_3

Day RE et al (2014) Human aquaporins: regulators of transcellular water flow. Biochem Biophys Acta 1840:1492-1506. doi:10. 1016/j.bbagen.2013.09.033

De Schutter K et al (2009) Genome sequence of the recombinant protein production host Pichia pastoris. Nat Biotechnol 27:561-566. doi:10.1038/nbt.1544

Eriksson S, Elbing K, Soderman O, Lindkvist-Petersson K, Topgaard $D$, Lasic S (2017) NMR quantification of diffusional exchange in cell suspensions with relaxation rate differences between intra and extracellular compartments. PLoS ONE 12:e177273. doi:10.1371/journal.pone.0177273

Fischer G et al (2009) Crystal structure of a yeast aquaporin at 1.15 angstrom reveals a novel gating mechanism. PLoS Biol 7:e1000130. doi:10.1371/journal.pbio.1000130

Geyer RR, Musa-Aziz R, Qin X, Boron WF (2013) Relative $\mathrm{CO}_{2} / \mathrm{NH}_{3}$ selectivities of mammalian aquaporins 0-9. Am J Physiol Cell Physiol 304:C985-C994. doi:10.1152/ajpcell.00033.2013

Hara-Chikuma M, Verkman AS (2008a) Aquaporin-3 facilitates epidermal cell migration and proliferation during wound healing. J Mol Med (Berl) 86:221-231. doi:10.1007/s00109-007-0272-4

Hara-Chikuma M, Verkman AS (2008b) Roles of aquaporin-3 in the epidermis. J Invest Dermatol 128:2145-2151. doi:10.1038/jid. 2008.70

Hedfalk K, Pettersson N, Oberg F, Hohmann S, Gordon E (2008) Production, characterization and crystallization of the Plasmodium falciparum aquaporin. Protein Expr Purif 59:69-78. doi:10. 1016/j.pep.2008.01.004

Ishibashi K, Kuwahara M, Gu Y, Tanaka Y, Marumo F, Sasaki S (1998) Cloning and functional expression of a new aquaporin (AQP9) abundantly expressed in the peripheral leukocytes permeable to water and urea, but not to glycerol. Biochem Biophys Res Commun 244:268-274. doi:10.1006/bbrc.1998. 8252

Kondo $\mathrm{H}$ et al (2002) Human aquaporin adipose (AQPap) gene: genomic structure, promoter analysis and functional mutation. Eur J Biochem/FEBS 269:1814-1826

Laforenza U, Bottino C, Gastaldi G (2016) Mammalian aquaglyceroporin function in metabolism. Biochem Biophys Acta 1858:1-11. doi:10.1016/j.bbamem.2015.10.004

Leung J, Pang A, Yuen WH, Kwong YL, Tse EW (2007) Relationship of expression of aquaglyceroporin 9 with arsenic uptake and sensitivity in leukemia cells. Blood 109:740-746. doi:10.1182/ blood-2006-04-019588

Liu Z, Shen J, Carbrey JM, Mukhopadhyay R, Agre P, Rosen BP (2002) Arsenite transport by mammalian aquaglyceroporins AQP7 and AQP9. Proc Natl Acad Sci U S A 99:6053-6058. doi:10.1073/pnas.092131899

Liu Z, Carbrey JM, Agre P, Rosen BP (2004) Arsenic trioxide uptake by human and rat aquaglyceroporins. Biochem Biophys Res Commun 316:1178-1185. doi:10.1016/j.bbrc.2004.03.003

Liu Y et al (2007) Aquaporin 9 is the major pathway for glycerol uptake by mouse erythrocytes, with implications for malarial virulence. Proc Natl Acad Sci U S A 104:12560-12564. doi:10. 1073/pnas.0705313104

Maeda N (2012) Implications of aquaglyceroporins 7 and 9 in glycerol metabolism and metabolic syndrome. Mol Asp Med 33:665-675. doi:10.1016/j.mam.2012.02.004

McDermott JR, Jiang X, Beene LC, Rosen BP, Liu Z (2010) Pentavalent methylated arsenicals are substrates of human AQP9. Biometals 23:119-127. doi:10.1007/s10534-009-9273-9

Muller-Lucks A, Gena P, Frascaria D, Altamura N, Svelto M, Beitz E, Calamita G (2013) Preparative scale production and functional 
reconstitution of a human aquaglyceroporin (AQP3) using a cell free expression system. N Biotechnol 30:545-551. doi:10.1016/j. nbt.2013.03.007

Ohgusu Y et al (2008) Functional characterization of human aquaporin 9 as a facilitative glycerol carrier. Drug Metab Pharmacokinet 23:279-284

Panaretou B, Piper P (2006) Isolation of yeast plasma membranes. Methods Mol Biol 313:27-32. doi:10.1385/1-59259-958-3:027

Pettersson N, Hagstrom J, Bill RM, Hohmann S (2006) Expression of heterologous aquaporins for functional analysis in Saccharomyces cerevisiae. Curr Genet 50:247-255. doi:10.1007/ s00294-006-0092-Z

Roudier N, Ripoche P, Gane P, Le Pennec PY, Daniels G, Cartron JP, Bailly P (2002) AQP3 deficiency in humans and the molecular basis of a novel blood group system, GIL. J Biol Chem 277:45854-45859. doi:10.1074/jbc.M208999200
Sohara E, Rai T, Miyazaki J, Verkman AS, Sasaki S, Uchida S (2005) Defective water and glycerol transport in the proximal tubules of AQP7 knockout mice. Am J Physiol Renal Physiol 289:F1195F1200. doi:10.1152/ajprenal.00133.2005

Tamas MJ et al (1999) Fps1p controls the accumulation and release of the compatible solute glycerol in yeast osmoregulation. Mol Microbiol 31:1087-1104

Ternes P et al (2011) Two pathways of sphingolipid biosynthesis are separated in the yeast Pichia pastoris. J Biol Chem 286:11401-11414. doi:10.1074/jbc.M110.193094

Verkman AS (2012) Aquaporins in clinical medicine. Annu Rev Med 63:303-316. doi:10.1146/annurev-med-043010-193843

Zeuthen T, Klaerke DA (1999) Transport of water and glycerol in aquaporin 3 is gated by $\mathrm{H}^{+}$. $\mathrm{J}$ Biol Chem 274:21631-21636 